\title{
Finessing a point: augmenting the core
}

\author{
Donald G. Saari • Garrett R. Asay
}

Received: 18 August 2008 / Accepted: 6 April 2009 / Published online: 21 April 2009 (C) The Author(s) 2009. This article is published with open access at Springerlink.com

\begin{abstract}
The "finesse point" introduced here extends the notion of a core; it is a position that minimizes what a candidate needs to do to counter moves that are made by an opponent. The definition, which is motivated by the "chaos theorem" as well as by the dynamics of positive and negative political campaigning, is also used to define a "malicious point," which is an optimal location from which a candidate can engage in "negative campaigning."
\end{abstract}

\section{Introduction}

McKelvey's (1979) seminal chaos theorem states that, with surprisingly relaxed conditions, an agenda can be created where it is possible to start at any specified proposal and, with a succession of majority votes, end at any other specified proposal. While examples exhibiting this troubling dynamic are easy to create, for the most part the extremes of McKelvey's theorem are not observed in practice. Why? Perhaps the typically more stable behavior is caused by an "incumbency effect" or other moderating forces. Contributing to our understanding of this more limited status is the finesse point (Saari 2007) that is developed here. As this point is explicitly designed to control the

Saari's research was supported by NSF grants DMI-0640817 and DMS-0631362. Earlier versions of these results were presented at a 9/2005 conference in Erice, Sicily (see Saari 2007), a 12/2005 IMBS conference, and several colloquia presentations. Our thanks for the useful suggestions that were made by two referees.

D. G. Saari $(\bowtie)$

Institute for Mathematical Behavioral Sciences, University of California,

Irvine 92697-5100, USA

e-mail: dsaari@uci.edu

G. R. Asay

Centers for Disease Control and Prevention, Atlanta, GA 30333, USA 
chaos theorem behavior, it can be expected to identify aspects of what permits more moderate behavior.

McKelvey's result centers around the existence of a core point; this is a position that no other alternative can beat with the specified voting rule. The power of the core is manifested by how it leads to valued concepts that help us understand elections and competitive interactions such as the well known "median voter theorem." The core, however, fails to exist in many natural settings; it is this non-existence that unleashes McKelvey's effect. Understandably, the negative conclusions that are associated with an empty core have encouraged a search for natural replacements. Examples include the uncovered set, yolk, and so forth. (Miller 2005 provides an excellent, informative survey.) The finesse point is a new contribution.

The idea motivating the finesse point is simple: if a core point cannot be beaten, then any extension, such as the finesse point, should be a point that minimizes what it takes to keep from being beaten. Stated in a game theoretic manner, the finesse point minimizes the needed effort to disrupt and destroy any minimal winning coalition (i.e., a coalition with just enough voters to ensure victory) that supports an opponent. After developing its properties, the finesse point is then related to the uncovered set, yolk, etc. Because the finesse point is designed to minimize what a candidate must do to attract enough of her opponent's supporters to avoid defeat, it is reasonable to treat it as the kind of position that a successful, experienced politician adopts to more easily respond to competitors. Indeed, the development of the finesse point borrows from apparent election dynamics such as positive and negative campaigning.

The finesse point also reflects a candidate's need to preserve a sense of credibility. Namely, changes in a candidate's position, or charges she makes about her opponent, cannot vary wildly. If, for instance, voters view negative campaigning as demonstrating malice toward an opponent, then adopting excessive stands would be counterproductive. To capture this sense, we define a "malicious point" as a stance from which charges cast by a candidate against her opponent can be minimal yet effective to ensure that her opponent's position is not a "winning" one. These finesse and malicious points are related as part of our main objective to find a position that minimizes the "effort" needed to control the consequences of the McKelvey chaos result.

\section{Basic ideas}

Standard notions of spatial voting are used where the "issue space" is represented by an Euclidean space $\mathbb{R}^{k}$ : each of the $k$ coordinate axes represents a separate issue. The components of a voter's ideal point, $\mathbf{p}=\left(p_{1}, \ldots, p_{k}\right)$, identify his "ideal" or "most preferred" outcome over these issues. As illustrated in Fig. 1a where bullets designate voters' ideal points, the dashed lines show that voter three's ideal position is to spend a moderate amount on domestic issues and a considerable amount on foreign aid. While our conclusions can be extended to include voter preferences that are represented with smooth, strictly convex utility functions, it suffices for our current purposes to assume that the closer a position is to a voter's ideal point, the more he likes it. These preferences are called "Euclidean." We adopt the standard assumption that no two ideal points agree. 


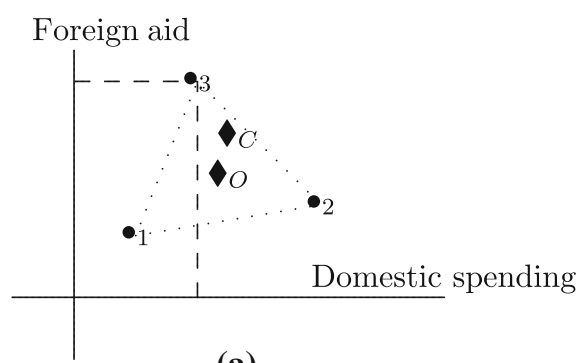

(a) $\dagger$

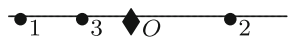

(b)

Fig. 1 Voter and candidate positions. a A two issue example, b a one issue example

Candidates' positions are also represented by points in issue space; they are the diamonds in Fig. 1a where the subscripts represent their names of "Candidate" and "Opponent." A voter prefers the candidate whose position is closer to his ideal point; e.g., in Fig. 1a, voter 1 prefers Opponent while voter 3 prefers Candidate. To add flexibility into applications and the discussion, treat a candidate's point as the voters' perception of her stance rather than her actual position.

This "perception" interpretation reflects the ever present problems of incomplete information and the objectives of negative campaigning. An illustration is the 1988 US presidential campaign where the "Americans for Bush" launched a "Willie Horton" ad, which featured a criminal who committed horrendous crimes while furloughed, to create the perception that the Democratic candidate Dukakis was "soft on crime." For purposes of the 1988 election, Dukakis' actual stance was not as consequential as the voters' perception. As this example also illustrates, we must expect there to be multiple perceptions of a candidate's position; e.g., members of one political party may perceive her stand quite differently than members of another party. Such differences, however, are ignored here primarily because it would detract from our main objective of introducing the finesse point. At each instant of time, then, assume there is a unique perception of each candidate.

The role of campaigning, whether positive or negative, is to change the voters' image of some candidate. For our purposes, positive campaigning is where a candidate's campaign tactics are directed toward changing her perception, or stance, with the voters. Negative campaigning is where her campaign tactics are directed toward changing the voters' views of her opponent.

Our main contribution is to extend the game theoretic concept of the core. In terms of an election, a candidate's position is a core point if it cannot be defeated by any position taken by her opponent. To illustrate with the majority vote and a single issue, the core agrees with the median voter's ideal point; e.g., in Fig. 1b, this is voter three's ideal point. To appreciate this well-known "Median Voter Theorem" (Downs 1957; Hotelling 1929), notice that if Opponent's position is anywhere else, as indicated by the diamond in Fig. 1b, Candidate can win by assuming a position closer to voter three's ideal point to attract the votes of voters one and three.

Obviously, each candidate would like to be positioned in the core. But if the core fails to exist, then a trailing candidate presumably seeks "weak spots" in an opponent's supporting coalition to encourage voters to change their vote. To motivate the finesse 
point, we first examine the possible reactions of a trailing candidate to any winning position of an opponent when a core does not exist, and how a winning candidate can make the campaign more difficult for her opponent.

\section{An empty core}

Our results hold for $q$-voting rules; this is where a winning candidate must receive $q$, the quota, or more of the $n$ votes, $\frac{n}{2}<q<n$. Let $\mathbb{C}(q, n)$ denote the set of $q$ rule core points for the specified voter preferences. For the simple majority vote, $q$ is the first integer greater than $\frac{n}{2}$, while for the two-thirds rule $q$ is the first integer greater than $\frac{2 n}{3} ; q$-rules often arise in politics (Nurmi 2002), even to elect a pope (Saari 1995 , 2007). We ignore the unanimity $q=n$ rule only because $\mathbb{C}(n, n)$ always exists (to find the issue spaces for which we can expect an empty $q$-rule core, see Saari 2004).

McKelvey's (1979) chaos result describes the troubling dynamics that can accompany an empty core. Choose any two positions in issue space represented by an initial $\mathbf{p}_{i}$ and a final $\mathbf{p}_{f}$. If a majority-vote core does not exist, then there exists a sequence of positions, $\mathbf{p}_{1}, \mathbf{p}_{2}, \ldots, \mathbf{p}_{m}$, where $\mathbf{p}_{1}=\mathbf{p}_{i}, \mathbf{p}_{m}=\mathbf{p}_{f}$, and each $\mathbf{p}_{j}$ is preferred by a majority of the voters over $\mathbf{p}_{j-1}$. Restated in words, with an empty core, it is possible to start anywhere and end up anywhere else via a carefully selected sequence of majority votes. Tataru $(1996,1999)$ extended McKelvey's seminal theorem to all $q$-rules: call this collection of results the "chaos theorem." Richards (1994) made this terminology appropriate by establishing a connection between the voting "chaos theorem" and "chaotic dynamics."

In simpler terms, the chaos theorem asserts that with an empty core, with sufficient time (required to carry out the necessary $m$ steps where $m$ may be a large integer) and skill in packaging successive positions, it is possible to lead the voters from any initial position to any desired final position - even if all voters view the final position as more undesirable than an earlier proposal! With Fig. 1a, for instance, there is a sequence of proposals starting at Opponent's initial position (the diamond near the center of the triangle) and, with a succession of majority vote victories, ending at the dagger located in the far upper right-hand corner of the figure. Clearly, all voters represented in Fig. 1a prefer the original position to the final one (for election campaigns, replace majority vote "victories" with majority vote "opinions").

Countering the wide and wild dynamics allowed by the chaos result, the finesse point introduces a centrally located position from which, with a candidate's thoughtful actions, a sense of stability is imposed. Namely, while the chaos theorem ensures that voters can be led from one position to any other, the finesse point is a stance from which only minimal modifications can respond to any proposed change. As indicated above, such a point can be loosely treated as the kind of position adopted by a successful, experienced politician; e.g., it might explain the incumbency effect.

To motivate the definition of the finesse point, we first determine the settings from which a candidate can use positive and/or negative campaigning. As asserted next with fairly general conditions, either approach can be successfully used at any moment, so the choice is determined by other factors. As both strategies always are viable, we must anticipate a mixture of positive and negative campaigning, which is what we 
observe. Incidentally, while three-voter results of this type can be easy to prove, the complexity significantly increases with the number of voters because of the added number of winning coalitions. What makes the proof of Theorem 1 immediate is the chaos theorem.

Theorem 1 For $n$ voters and any number of issues in a two candidate q-rule election, suppose that $\mathbb{C}(q, n)$ is empty. For any position of an opponent who currently is winning, the losing candidate can adopt a winning position by using either positive or negative campaigning.

The chaos theorem ensures a sequence of positions $\mathbf{p}_{i}, \ldots, \mathbf{p}_{f}$ starting at an initial $\mathbf{p}_{i}$, which we select to be the opponent's current position, and ending at a final $\mathbf{p}_{f}$, where each position is preferred by $q$ of the voters over the preceding one. A candidate's positive winning strategy is to adopt a position that beats $\mathbf{p}_{i}$, the opponent's current position. The chaos theorem ensures for any $q$-rule, any number of voters, and any number of issues, that such a position always exists. As the candidate's own perceived position is changed, this strategy is positive campaigning.

To prove that a negative strategy always exists, choose the initial position $\mathbf{p}_{i}$ as the opponent's current position, and let the final position $\mathbf{p}_{f}$ be a specific candidate's current position. According to the chaos theorem, there exists a position that makes $\mathbf{p}_{f}$ a winning position. Consequently, the candidate's negative campaigning strategy is to change her opponent's perceived stance to one of the guaranteed settings that the candidate can beat.

\subsection{An illustrating example}

Before illustrating Theorem 1 with the three-voter Fig. 1a preferences, notice that the Pareto set (i.e., positions that if changed in any manner will hurt some voter) consists of the points inside the convex hull defined by the voters' ideal points; this is the triangle with vertices defined by the three ideal points. In Fig. 1a, both candidates have their positions inside the Pareto set while the dagger is not.

With Euclidean preferences, voters one and two vote for Opponent to make her the majority vote winner. The options for Candidate to successfully react are determined by her winning set (Shepsle and Weingast 1984). To find this set depicted in Fig. 2a, draw a circle about each ideal point that passes through Opponent's position; ${ }^{1}$ as all points inside this circle are closer to the voter's ideal point, he prefers them to Opponent's position. The resulting trefoil is Candidate's winning set; namely, any point in a leaf is preferred by a majority of the voters over Opponent's position. The lower leaf, for instance, identifies all positions that voters one and two prefer to Opponent's perceived opinion. But Candidate's current position is not in the Fig. 2a trefoil, so Opponent is winning. Clearly, Candidate's positive campaigning strategies move her new stance inside the trefoil. Presumably the easiest approach (i.e., involving the

\footnotetext{
1 This set, which denotes all positions that beat Opponent, often is denoted by $\mathcal{W}(O)$. With two candidates, it is more intuitive to call this "Candidate's winning set" because it identifies positions where Candidate can win. When discussing legislative actions, the traditional notion is preferred.
} 


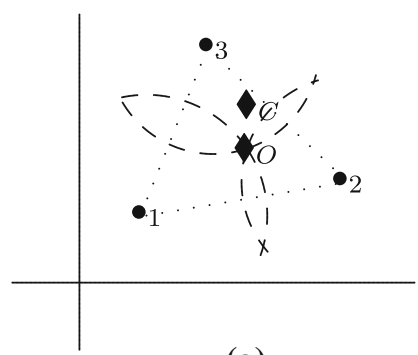

(a)

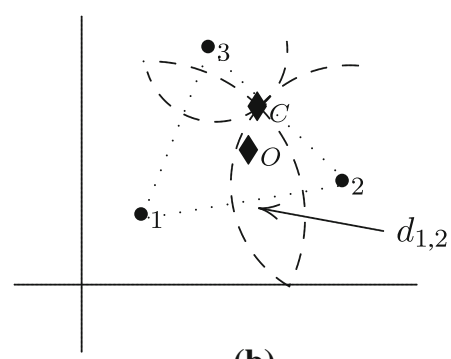

(b)

Fig. 2 Strategic options for Candidate. a Positive campaigning, b negative campaigning

least change) is to select a position directly toward voter two's ideal point: doing so moves Candidate's newly perceived beliefs into the leaf on the upper right-hand side to solidify her support from the $\{2,3\}$ winning coalition. In other words, relative to Opponent's current position, $\{2,3\}$ is the closest winning coalition for Candidate.

Candidate's choices can also be described with Opponent's winning set as depicted in Fig. 2b. Pass circles through Candidate's perceived position: as Opponent is positioned in the trefoil's lower leaf, she currently is winning. The optimal way for Candidate to "redefine" her own position and drop Opponent's position out of this winning set is to move directly toward voter two's ideal point (i.e., emphasize these issues); as this change reduces the radius of the circle centered at voter two's ideal point, it moves the winning set away from Opponent's position.

To illustrate Candidate's negative campaigning choices relative to Opponent's winning set in Fig. 2b, her strategies must move Opponent's perceived position outside of this set. By using the closest leaf edge of this example, this objective is most easily accomplished by painting Opponent's views as being sufficiently to the left-directly away from voter two's ideal point.

\subsection{Options for the winning candidate}

The leading candidate, Opponent in Fig. 2, also has negative and positive campaigning opportunities, which motivate what we will call the "selective finesse point" in Sect. 6. By currently winning, Opponent's position is in her winning set depicted in Fig. 2b. She may wish to make Candidate's task more difficult by increasing both the size of the leaf in which her position is located and the minimal distance of her position from the leaf's boundary. This can be done by increasing the radius to voter two's ideal point; i.e., increase the distance of Candidate's perceived position from the ideal points in Opponent's majority coalition $(\{1,2\}$ in Fig. 2 a). This negative campaigning assumes the flavor of "Candidate is not representing the views of the majority consisting of voters one and two," or "Candidate's views are more representative of voter three than any other voter." Contrary to the occasionally suggested impression, negative campaigning is a viable option for a winning candidate, and it is observed in actual elections. 
Positive campaigning options are determined by Fig. 2a. Opponent must adopt a position to move her position so that Candidate's winning set is moved farther from Candidate's current perceived position; i.e., reduce the distance from Opponent's position to the ideal points of voter's one and two (with a slight emphasis on voter two) to better reflect the preferences of her winning coalition. Using Fig. 2b, her positive strategy moves her position so that she is better identified with her winning coalition. These are commonly observed strategies.

\subsection{Costs of negative and positive campaigning}

With an empty core and both candidate's positions in the Fig. 2 Pareto set, adopting either a negative or a positive approach could change Candidate's fortunes. Which approach involves a smaller change in perception? The answer follows.

Theorem 2 With three voters and two candidates in a majority vote election with an empty core where voters have Euclidean preferences, if the campaign costs are measured by the shortest distance to a winning position and if both candidates are in the Pareto set, then a positive and a negative strategy are equally expensive for the trailing candidate.

In words, when measured by the required "distance" to change voters' perceptions, neither positive nor negative campaigning has an advantage over the other. This equality has pragmatic significance because actual campaign costs (measured by money, public opinion, etc.) per unit change of perception differ between positive and negative strategies. This differing cost per unit change determines the least expensive way to attract supporting voters; e.g., if negative campaigning attracts free press coverage or publicity, then it is cheaper to campaign negatively.

The intuition for the proof (Appendix) is central our definition of the finesse point; it captures the objective of persuading "swing voters" to leave and disrupt an existing minimal winning coalition (favoring an opponent) to avoid being beaten. The ultimate goal, of course, is to win, so the swing voters may help to create a new minimal winning coalition (favoring the candidate). However, "winning" is not necessary to achieve our goal of extending the core. Namely, for the same reasons why core points are defined to avoid being beaten, but not necessarily winning, the finesse points are designed to minimize the needed effort to avoid being beaten. (For the majority vote, the difference arises only when there are an even number of voters.)

To illustrate the "swing voter" comment with Fig. 2, voter two is the swing voter for both of Candidate's strategies, and voter three is the target for both of Opponent's strategies. In Fig. 2a, a positive campaign by Candidate must be directed toward voter two to encourage him to leave the winning $\{1,2\}$ coalition and create the winning $\{2,3\}$ coalition; in Fig. 2 b, a negative campaign also is targeted toward voter two to destroy Opponent's winning $\{1,2\}$ coalition. The amount of change needed for each strategy to achieve a winning coalition is the shortest distance from the targeted perceived position to the leaf edge defined by the swing voter's ideal point. As the edges are circles centered at ideal points, the distances are determined by the radii of the 
different circles. The proof of Theorem 2 shows that the nearest distance is the same for a positive or negative approach.

\section{Finesse points}

A candidate strives to attract a winning coalition. But a winning coalition contains minimal ones, so we emphasize these structures. To be precise, a minimal winning coalition for a $q$-rule consists of precisely $q$ voters where the convex hull defined by their ideal points does not contain any other voters' ideal points. By being "minimal," the coalition's "winning status" is lost should even a single voter leave the coalition; this permits the analysis to concentrate on individual preferences.

With any number of voters and issues, we need to find a position that minimizes the amount of change (for positive and/or negative campaigning) to react to any minimal winning coalition of Opponent; it minimizes what is needed to convert some member of any minimal winning coalition supporting Opponent to join a coalition that supports Candidate's new position. By moving a voter out of Opponent's minimal winning coalition, the coalition no longer is winning, so Candidate avoids defeat (but, as true with the core points, she may not be the winner). How this can be done depends on what a candidate knows about her opponent. If, for instance, her opponent must support certain beliefs (e.g., a specific position on social security or abortion rights), then certain coalitions are irrelevant while others gain in importance.

Start with the worse case scenario where a candidate has no knowledge about her opponent. (With legislative agenda models, this corresponds to having no prior knowledge about possible counterproposals.) With no information, the approach must involve all possible minimal winning coalitions. Settings involving more information are described in Sect. 6.

As suggested by Fig. 2, the worse case scenario facing the positive campaigning of Candidate is if Opponent's position is precisely at the middle of the widest portion of the biggest leaf of the winning set. To avoid defeat, Candidate must woo at least one of these voters to join her coalition.

Stated geometrically, Candidate must change her position to entice some voter to leave Opponent's winning coalition to join a coalition that supports Candidate. The geometry shows that while this change must be targeted toward specific swing voters, the amount of change is no more than half the leaf's width. ${ }^{2}$ To find this region, for a winning coalition $\mathcal{C}$, denote its Pareto set by $\mathcal{P}(\mathcal{C})$. (That is, $\mathcal{P}(\mathcal{C})$ is the set of points where any change causes some coalition member to have a poorer outcome. With Euclidean preferences, $\mathcal{P}(\mathcal{C})$ is the convex hull of the ideal points of a coalition; e.g., with two voters it is a line connecting the two ideal points.) The widest portion of a leaf is where it intersects $\mathcal{P}(\mathcal{C})$; this is the interval defined by the intersection of a leaf with the $\mathcal{C}$ edge of the triangle.

Let $d_{C}$ be the width of the appropriate leaf of the winning set defined by the winning coalition $\mathcal{C}$; e.g., in Fig. 2 b, $d_{\{1,2\}}$ is the length of the interval on the triangle's lower

\footnotetext{
2 Remember, the worse case is if the opponent's position is at the middle of the widest portion, so the necessary change in a radius is this distance, which is half the leaf's width.
} 
leg that also is in the lower leaf of the winning set. Using the radii of circles in the figure, $r_{j}$, and the length of the segment between the $i$ th and $j$ th ideal points, $z_{i, j}$, it follows that

$$
d_{i, j}=r_{i}+r_{j}-z_{i, j}
$$

The $d_{\{2,3\}}<d_{\{1,3\}}<d_{\{1,2\}}$ values of Fig. $2 \mathrm{~b}$ benefit Candidate if Opponent courts the $\{2,3\}$ coalition, but Candidate's Fig. $2 b$ position would create problems if Opponent decided to court the $\{1,2\}$ group. The reason is that as $d_{\{2,3\}}<d_{\{1,2\}}$, necessary changes in Candidate's position to attract a voter from $\{2,3\}$ (the change is bounded by $\frac{d_{\{2,3\}}}{2}$ ) are more modest than if Candidate had to attract a voter from $\{1,2\}$. If there is no information about an opponent, then the goal is to minimize the maximum $d_{C}$ value over all possible minimal winning coalitions. This is accomplished with the finesse point.

\subsection{A $d$-finesse point}

To introduce a " $d$-finesse point" for three voters (Saari 2007), recall from high school geometry that an ellipse can be created as indicated in Fig. 3a. About two pegs on a board place a string (given by the dashed line) tied in a loop with a length larger than twice the distance between pegs. Put a pencil inside the string and trace it around creating the dotted curve: this curve is an ellipse. Call it a " $d$-ellipse" where $2 d$ is the extra length of the string; i.e., $2 d$ equals the length of the string minus twice the length between the pegs. Equivalently (because the part of the string connecting the pegs is taut),

$2 d$ is the sum of the lengths of the two dashed slanted lines (the two radii from the pegs to the point) minus the length between the pegs.

By comparing this definition with Eq. 1 and the definition of $d_{C}$, it follows that all points on the $d$-ellipse defined by two ideal points have $d_{C}=2 d$ for this two-voter coalition. In higher dimensions (i.e., with more issues), this construction creates a $d$-ellipsoid. (an alternative definition for the $d$-ellipse is given below to motivate the construction of the "generalized ellipsoid").

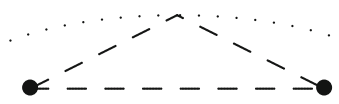

(a)

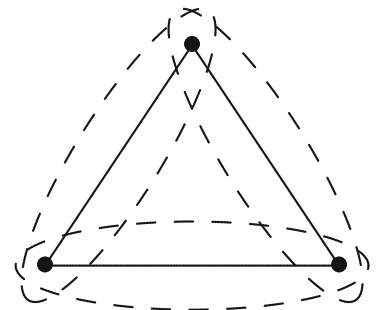

(b)

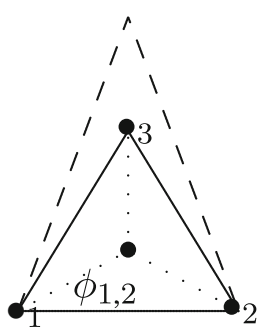

(c)

Fig. 3 Finding the $d$-finesse point. a Creating an ellipse, $\mathbf{b}$ intersecting ellipses, $\mathbf{c}$ moving the finesse point 


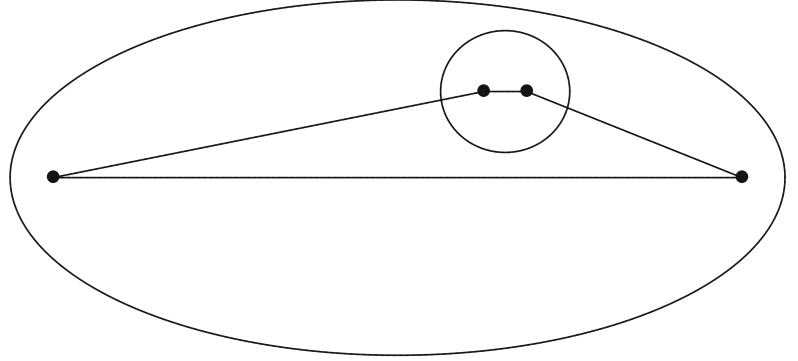

(a)

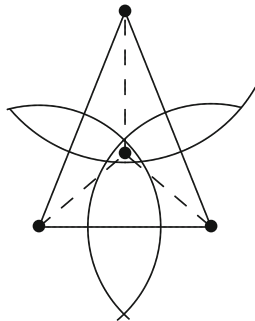

(b)

Fig. 4 Structure of sets. a Two of the six $E_{d}^{2}(C)$ ellipses, $\mathbf{b}$ winning set for thee issues

For the majority vote and three voters a minimal winning coalition consists of any two ideal points. About each coalition, construct a $d$-ellipse. For any two coalitions, these two $d$-ellipses intersect at a point in the triangle. From this point and with either of these two coalitions, the widest portion of an opponent's winning set leaf is precisely $2 d$. But if this point is not in the $d$-ellipse for the third coalition, then the widest portion of the opponent's winning set for the third coalition exceeds $2 d$. Thus, if an opponent courted this third coalition, it would create problems. To avoid these problems, the " $d$-finesse point" is where all three ellipses first intersect in a unique point: this point has the smallest $d$ value over all winning coalitions. In other words, once positioned at this point, a change of no more than $d$ will disrupt an opponent's winning position.

To extend this construction to any number of voters, any specified $q$-rule, with only two issues, start with a minimal winning coalition $\mathcal{C}$. Let $E_{d}^{2}(\mathcal{C})$ be the union of $\mathcal{P}(\mathcal{C})$ and all $d$-ellipsoids (interior and surface) defined by each pair of ideal points in $\mathcal{C}$ (see Fig. 4a). To explain why $\mathcal{P}(\mathcal{C})$ is included in $E_{d}^{2}(\mathcal{C})$, recall that the finesse point minimizes what it takes to keep from being beaten by a coalition. Namely, $E_{d}^{2}(\mathcal{C})$ identifies all possible points for which an appropriate change of no more then $d$ will ensure that the candidate cannot be beaten by the voters in coalition $\mathcal{C}$. As this property clearly holds if our candidate's point is in $\mathcal{P}(\mathcal{C}), \mathcal{P}(\mathcal{C})$ must be in $E_{d}^{2}(\mathcal{C})$.

To explain this construction and to indicate how to identify which voters from coalition $\mathcal{C}$ a candidate should target her appeal, notice that each $d$-ellipsoid is defined by a pair of voters from $\mathcal{C}$; for any proposal $\mathbf{p}$ on this ellipsoid, it takes an appropriate change of no more than distance $d$ to move one of these swing voters out of coalition $\mathcal{C}$; the voters that remain no longer constitute a minimal winning coalition for Opponent. If a point $\mathbf{p}$ is on the boundary of $E_{d}^{2}(C)$, but not in $\mathcal{P}(\mathcal{C})$, then $\mathbf{p}$ is on the $d$-ellipsoid boundary for some two ideal points from $\mathcal{C}$; the width of the lens defined by $\mathbf{p}$ and these two ideal points is $2 d$. If Candidate's position is at $\mathbf{p}$ and if $\mathcal{C}$ is a winning coalition supporting Opponent, then at least one the voters defined by these two ideal points is the swing voter that should be the target of Candidate's changed position.

For all other pairs of ideal points in $\mathcal{C}$, if its $d$-ellipsoid does not include $\mathbf{p}$, then the lens width from $\mathbf{p}$ is wider than $2 d$. As the winning set is in the intersection of all lens, $2 d$ is an upper bound on the width of the winning set. Thus if $\mathcal{C}$ is a minimal winning coalition supporting Opponent, then it is possible to alter $\mathbf{p}$ by no more than distance $d$ to change the allegiance of one of the $\mathcal{C}$ voters away from Opponent. Clearly, this 
change in $\mathbf{p}$ must be aimed toward one of the two voters in the $d$-ellipsoid boundary that contains p; i.e., this $d$-ellipsoid identifies which voters to target. The following definition mimics the ellipse construction.

Definition 1 For two issues, a q-rule $d$-finesse point is a point that is in each $E_{d}^{2}(\mathcal{C})$ for all minimal winning coalitions $\mathcal{C}$ where $d$ is the smallest possible value permitting such a point to exist. The adjustment region, denoted by $\mathbb{S}_{d}^{2}(q)$, is a ball centered about a $d$-finesse point with radius $d$.

As shown in the Appendix, the $E_{d}^{2}(C)$ structures describe the winning set's width with two issues. However, ellipsoids no longer are appropriate with three or more issues. In Fig. 4b, for instance, suppose for a $q=3$ rule that a proposal $\mathbf{p}$ is above the triangular $\mathcal{P}(\mathcal{C})$ where the coalition is represented by the three vertices (for now, ignore the center bullet). To find the winning set, for each voter, a sphere centered at the voter's ideal point is constructed so that $\mathbf{p}$ is on its boundary. Because the winning set has to appeal to all members in coalition $\mathcal{C}$, it is the $\mathcal{P}(\mathcal{C})$ region that is in each of the three spheres. As such, with more issues, the winning set is the small triangular shaped region in the interior, rather than the lenses. A worse case scenario positions Opponent at the bullet in the center of this triangular region; this position is $\alpha$-units (for some $\alpha$ value) from each of the three surfaces. But the size of this triangular region is quite small compared to the width of any of the three lens. So, if ellipses were used for an extension of $E_{d}^{2}(C)$ for three issues, we would capture the widths of the lens rather than the much smaller, appropriate $\alpha$ value. A new construction is needed.

To describe the approach, first consider an alternative way to construct the $d$-ellipses. For the $i$ th and $j$ th ideal points, select any point on the line interval $\mathcal{P}(\{i, j\})$. Denote the distances from the selected point to the ideal points as $\rho_{k}, k=i, j$. The points of intersection of the circles of radius $r_{k}=\rho_{k}+d$ for $k=i, j$, are two points on the $d$-ellipse; the center of the lens defined by the $r_{j}$ circles is the selected $\mathcal{P}(\{i, j\})$ point. The $d$-ellipse is created by varying the selected point in $\mathcal{P}(\{i, j\})$.

Similarly, for $k$ issues and $k$ ideal points that define a $(k-1)$-dimensional simplex, define the generalized $k$-fold $d$-ellipsoid for this coalition $\mathcal{K}$ in the following manner: Select any point in $\mathcal{P}(\mathcal{K})$; it uniquely defines $k$ distances, $\rho_{j}$, to each ideal point. Each $r_{j}=\rho_{j}+d$ defines a sphere about the $j$ th voter's ideal points; points on the intersection of all $k$ spheres about the $k$ ideal points are on the generalized ellipsoid (the intersection of these spheres replaces the leafs from the earlier discussion). The generalized ellipsoid is generated by carrying out this construction over all $\mathcal{P}(\mathcal{K})$ points. As the $\rho_{j}$ 's define the center point of the curvilinear region defined by the $r_{j}$ 's, any point in this curvilinear region is at most $d$-distance from the nearest surface of a sphere (or, from the generalized lens).

A "generalized ellipsoid" is not an ellipsoid; i.e., it does not satisfy an ellipsoid's usual quadratic equations. ${ }^{3}$ The construction of this surface is illustrated with Fig. 4b, where the three ideal points define the vertices of a triangle. Select any point in this plane; e.g., the bullet in the center. The lengths of the dashed lines from each vertex to the selected point define the three $\rho_{j}$ values. The associated points on the generalized

\footnotetext{
3 While we have not computed the equations for the surface, it is an algebraic expression of degree no more than $2(k-1)$.
} 
ellipsoid surface are determined by the three spheres of radius $r_{j}=\rho_{j}+d$ that are centered about the respective ideal points. By geometry, these three spheres meet in two unique points; one is above the plane and the other is below (a line drawn between the two defined points is orthogonal to the plane and passes through the selected point). The generalized ellipsoid is created by moving the bullet over all points in the triangle. The arcs in the figure are where the spheres, or the voters' level sets, intersect the plane; they define a portion of the win set boundary (the rest of the win set, the intersection of the spheres, is above and below the plane).

The points defining the generalized ellipsoid in Fig. $4 \mathrm{~b}$ are directly above and below the simplex. The value of $d$ (equals $\alpha$ ) is smaller than what would be possible if we had used actual ellipsoids defined by pairs of points. The reason is that the three-fold $d$-value is determined by the distance directly above the center point of the curvilinear triangle, while for an ellipsoid, a significant part of its $d$ value involves reaching the center point of the curvilinear triangle before moving above it. This extra effort, creates an incorrect and overly large $d$ value.

Definition 2 For a $q$-rule and a minimal winning coalition $\mathcal{C}$, let $E_{d}(\mathcal{C})$ be the union of $\mathcal{P}(\mathcal{C})$, the $d$-ellipsoid for each pair of ideal points in $\mathcal{C}$, and the generalized $k$-fold $d$-ellipsoid for each set of $k \geq 3$ ideal points in $\mathcal{C}$ that define a $(k-1)$ dimensional simplex, $k=3, \ldots, n$. A $q$-rule $d$-finesse point is a point that is in each $E_{d}(\mathcal{C})$ for all minimal winning coalitions $\mathcal{C}$ such that $d$ is the smallest possible value allowing such a point to exist. The adjustment region, denoted by $\mathbb{S}_{d}(q)$, is a ball centered about a $d$-finesse point with radius $d$.

Some results follow; proofs are in the Appendix.

Theorem 3 For a q-rule with any finite number of voters and issues, the following are true.

1. A q-rule $d$-finesse point always exists.

2. A point is a q-rule $d$-finesse point for $d=0$ if and only if it is a q-rule core point.

3. A q-rule $d$-finesse point is a q-rule malicious point. The maximum amount of change in a position needed to respond to the opponent, with either positive or negative campaigning, is $d$; it is a change in the adjustment region $\mathbb{S}_{d}(q)$.

The $d$-finesse point achieves our goal; not only is it a natural generalization of the core (part 2), but the $d$ value indicates how far the coalitions are removed from defining a core point.

This connection with the core yields other conclusions. For instance, as a core point need not be unique, the $d$-finesse points (Theorem 3), or the malicious points, need not be unique. A four-voter illustration is where all ideal points are on a line, so the majority vote core is the subinterval between the second and third voters' ideal points.

Part 3 asserts that the $d$-finesse point and malicious point agree. At this common point the necessary change is no more than $d$ for negative or positive campaigning, so it is in $\mathbb{S}_{d}(q)$. Thus Theorem 3 extends Theorem 2 to any number of voters, issues, and $q$-rules. By specifying the amount of change required to counter any action, $d$ provides a measure of the inherent stability, or instability, of the system. Namely, "small" $d$-values suggest a fairly stable system, while "large" $d$-values can require more extreme changes to avoid the chaos theorem consequences. 


\section{Geometry of the finesse point}

To develop intuition about the finesse point, start with the three voter setting where the geometry is easier to understand. Let $r_{j}$ be the distance from the $j$ th voter's ideal point to the finesse point and $z_{j, k}$ the distance between the $j$ th and $k$ th voters' ideal points. Changes in voter preferences-their ideal points-change the $z_{j, k}$ values. The goal is to discover the corresponding changes in the location of the finesse point, as given by the $r_{j}$ and $d$ values. It is interesting (Eq. 2) to discover how changes in, say, $r_{1}$ and $r_{3}$, are governed by the positioning of the second voter's ideal point.

Theorem 4 Let the three voters be denoted by $i, j, k$. The position of the finesse point and the value of $d$ satisfy the following expressions.

$$
\begin{gathered}
r_{j}-r_{k}=z_{j, i}-z_{k, i} \\
d=r_{j}-\frac{1}{2}\left[z_{j, k}+z_{j, i}-z_{i, k}\right]=\frac{1}{3} \sum_{j} r_{j}-\frac{1}{6} \sum_{j<k} z_{j, k} .
\end{gathered}
$$

According to Eq. 2, when the ideal points define an equilateral triangle (solid lines of Fig. 3c) where $z_{1,2}=z_{2,3}=z_{1,3}$, then $r_{1}=r_{2}=r_{2}$ and the $d$-finesse point (the bullet) is at the triangle's center. The $d$ value is surprisingly small; e.g., elementary trigonometry proves that $r_{1}=\frac{\sqrt{3}}{3} z_{1,2}$; by using Eq. 3, $d$ is about $0.0773 z_{1,2}$, or less than $8 \%$ of the common distance between ideal points (in an example in the Appendix (Eq. 10), the $d$ value is about $2.6 \%$ of the length of the shortest leg).

Moving the top ideal point, which belongs to voter 3, directly upwards (dashed lines in Fig. 3c) creates an isosceles triangle, where $z_{1,3}=z_{2,3}$, so $r_{1}=r_{2}$ while $r_{3}=r_{1}+\left(z_{2,3}-z_{1,2}\right)$. (From Eq. 2, differences in leg lengths define differences in $r_{j}$ lengths.) Direct trigonometric computations (Appendix) prove that the common $r_{1}=r_{2}$ value increases, and the finesse point moves upwards. But no matter how far the ideal point moves upwards, the inequality $\frac{z_{1,2}}{2}<r_{1}<z_{1,2}$ always is satisfied! Consequently, the finesse point remains near the shorter edge. Also, from, Eq. 3 we have that

$$
d=\frac{1}{3}\left[3 r_{1}+\left(z_{1,3}-z_{1,2}\right)\right]-\frac{1}{6}\left[3 z_{1,2}+2\left(z_{1,3}-z_{1,2}\right)\right]=r_{1}-\frac{1}{2} z_{1,2},
$$

which, because $r_{1}<z_{1,2}$, means that $d<\frac{z_{1,2}}{2}$, or less than half the shortest leg. (It is much smaller.)

In the the portion of the Appendix that follows the proof of Theorem 4, we develop an easy algebraic way to find the $r_{j}$ and $d$ values, along with the finesse point's position, for all possible three-voter majority vote settings. But to develop intuition about what can happen, it is informative to use Theorem 4 to appreciate how changes in the ideal points change the finesse point. Let $\phi_{k, j}$ be the angle with vertex at the $k$ th voter's ideal point defined by the legs $r_{k}$ and $z_{k, j} ; \phi_{1,2}$ is depicted in Fig. 3c (so, $\phi_{k, j}$ and $\phi_{j, k}$ are different angles). 
Theorem 5 Assume that $z_{1,2}<z_{1,3}<z_{2,3}$; i.e., the two shorter legs of the triangle are attached to voter one's ideal point, and the two longer legs are attached to voter three's ideal points. Then

$$
r_{1}<r_{2}<r_{3}, \quad \text { and } \phi_{1,2}<\phi_{1,3}, \quad \phi_{2,1}<\phi_{2,3}, \quad \phi_{3,1}<\phi_{3,2} .
$$

It follows, again, that the finesse point is closest to the ideal point with the shorter legs and farthest from the one with the longer legs. Stated in words, the finesse point is closer to a coalition with more coherent views than one with separated views. (Indeed, $r_{j}$ is smaller than the shorter leg connecting the $j$ th voter's ideal point to some other voter's point, and $d$ is strictly less than half the length of the shortest leg. When $z_{j, k}$ leg lengths agree, appropriate $r_{j}$ and $\phi_{j, k}$ inequalities become equalities. ${ }^{4}$ ) As $\phi_{j, k}+\phi_{j, i}$ defines the triangle's angle at vertex $j$, it follows from Eq. 4 that about each ideal point, the finesse point's position must be skewed toward the shorter leg.

As the number of voters increases, the $E_{d}(\mathcal{C})$ structure provides surprises because the $d$-ellipse for a larger $z_{j, k}$ value is fatter (this "fatness" explains the Theorem 5 assertion that the finesse point is farther from the most distant ideal point; it also permits the relatively small $d$ values for the finesse point). Indeed, using the equation for an ellipse, it follows that (proof is in the Appendix)

$$
\text { the widest portion of a } d \text {-ellipse for } z_{j, k} \text { is } 2 d \sqrt{1+\frac{z_{j, k}}{d}} \text {. }
$$

Small $z_{j, k}$ values represent closely positioned ideal points, here (Eq. 5) the ellipse resembles a circle where the widest portion is slightly larger than the circle's diameter, or $2 d$. But if $z_{j, k}=3 d$, the width is $4 d$, and if $z_{j, k}=24 d$, the $d$-ellipse width grows to $10 d$. As a consequence, the $d$-ellipse of a pair of $\mathcal{C}$ points that are sufficiently far apart can be the $E_{d}(\mathcal{C})$ portion that defines the finesse point; this holds even if this pair ends up being the most distant of $\mathcal{C}$ points from the finesse point!

This assertion is dramatically illustrated in Fig. 4 a with the four-voter minimal coalition (as $\mathcal{C}$ is a minimal winning coalition for a $q=4$ rule, $n$ could be 5 , 6, or 7 ). With four ideal points, $E_{d}(\mathcal{C})$ has six ellipses; only the two extreme ones are displayed. The $d$ value used for this figure is the distance between the two closest ideal points. While these two ideal points may be closer to the eventual position of the finesse point, the properties of $E_{d}(\mathcal{C})$ and the location of the finesse point can be governed by the $d$-ellipse defined by the two most separated ideal points; they represent both intuitively and mathematically a "weak spot" (i.e., possible swing voters) in a coalition supporting an opponent. The actual location of the finesse point depends on the positions of all other minimal winning coalitions.

Notice how this construction leads to assertions that corresponds to intuition: If Candidate wants to siphon voters away from $\mathcal{C}$ that supports Opponent, an optimal way is to consider those members whose beliefs are weak with respect to the rest of $\mathcal{C}$; these points can define the largest $z_{j, k}$. Whether this strategy holds depends on

\footnotetext{
${ }^{4}$ Certain Eq. 4 inequalities reverse with changes in leg lengths; they become equalities when leg lengths agree.
} 
whether the $z_{j, k}$ leg creates the appropriate $E_{d}(\mathcal{C})$ ellipse that meets Candidate's current position. Also, expect a finesse point to be closer to a compact minimal winning coalition, which suggests that this is a group with a cohesion in beliefs, than to a coalition with spread apart ideal points, which indicates a coalition with a diversity in beliefs.

\subsection{Comparison with the yolk, uncovered set, etc}

Ferejohn et al. (1984) defined the yolk to be the sphere of minimal radius that intersects all median lines. Here a median line is the natural extension of the median point: it is a line (hyperplane in higher dimensions) with no more than half of the voters on either side. The yolk extends, in an obvious manner, to the "q-rule yolk."

Definition 3 For $\frac{n}{2}<q<n$, a $q$-line is a line (or hyperplane) with less than $q$ of the voters' ideal points on either side. A $q$-rule yolk, $\mathbb{Y}(q)$, is the circle (or sphere) of minimal radius that intersects all $q$-lines.

A median line divides the space into two parts where no winning coalition is strictly on either side. This means that a winning coalition must include ideal points from, or across, the median line. As a core point must be on all median lines, the yolk is an extension of the core. Similarly, the $q$-hyperplane divides the space so that no $q$-vote winning coalition is strictly in either portion. For all of the examples that we have investigated, the finesse point's adjustment region $\mathbb{S}_{d}(q)$ sharply refines the yolk. Our next result identifies some of these situations. With the equilateral triangle of Fig. 3c, for instance, the yolk's center is at the finesse point. The yolk's radius is 0.25 the length of a triangle edge while, as shown above, $d$ equals only about 0.077 of the edge length.

We expect, but have not proved, that $\mathbb{S}_{d}(q) \subset \mathbb{Y}(q)$ always holds. This expression is true, for instance, if all of the ideal points are on the Pareto set's boundary, but a general result has eluded us due to the complexity of the yolk as demonstrated by Stone and Tovey (1992) (see also Miller 2005).

Miller (1980) introduced, and McKelvey (1986) further developed, the uncovered set for the majority vote. Again, the extension of this notion to $q$-rules is immediate. Namely, position $\mathbf{p}$ is covered by $\mathbf{p}_{1}$ with the $q$-rule if $\mathbf{p}_{1}$ beats $\mathbf{p}$ and every point that beats $\mathbf{p}_{1}$ also beats $\mathbf{p}$. The $q$-rule uncovered set consists of all points that are not covered by any other point. As a covered proposal probably would not be a legislative outcome, the $q$-vote uncovered set identifies plausible $q$-vote legislative outcomes. Thus another favorable property of the $q$-rule $d$-finesse point is that it is in the $q$-rule uncovered set.

Wuffle et al. (1989) introduced a "finagle point," which is intended to minimize what it takes for a candidate to win with the majority vote. Whenever "not losing" with the majority vote is equivalent to "winning," such as with an odd number of voters, the finagle point should agree with the finesse point. But the complicated definition and construction of the finagle point are hard to follow, so it has been defined only for two issues, the majority vote, and with three voters, or for special two-issue settings involving an odd number of voters where their ideal points define a highly symmetric 
configuration. While there is no natural extension of the finagle point even for standard situations involving, say, four or five voters with an arbitrary positioning of their ideal points, we prove for at least for three voters, two issues, and the majority vote that the finagle point agrees with the finesse point (because the finagle point agrees with the finesse point in this setting, our construction of the finesse point provides an easier way to construct the finagle point).

Theorem 6 For $n \geq 3$ voters, we have the following:

1. With $n-1$ issues and $n$ ideal points that define $a(n-1)$-dimensional simplex, $\mathbb{S}_{d}(q)$ is a proper subset of $\mathbb{Y}(q)$; the yolk's center is in $\mathbb{S}_{d}(q)$. Thus, with three voters the majority-vote adjustment set is a proper subset of the yolk and the yolk's center is in the adjustment set.

2. A q-rule finesse point generally is not at the center of $\mathbb{Y}(q)$. Indeed, for $n=3$, the d-finesse point is at the center of $\mathbb{Y}(2)$ iff the ideal points define an equilateral triangle.

3. If $d(q)$ is the $d$-value for a $q$-rule finesse point and if $q_{1}<q_{2}$, then $d\left(q_{2}\right) \leq d\left(q_{1}\right)$.

4. A q-rule d-finesse point is a q-rule uncovered point.

5. For three voters, two issues, and the majority vote, the finesse point agrees with the finagle point.

As part 1 shows, the adjustment set can be a (sharp) refinement of the yolk.

Part 3 asserts, as we should expect, that with a stronger $q$ rule, the amount of change can be smaller. But while $\mathbb{S}_{d}\left(q_{2}\right) \subseteq \mathbb{S}_{d}\left(q_{1}\right)$ holds for all examples that we have investigated, this relationship has not been established in general.

A combination of parts 1 and 5 answers a Wuffle et al. (1989) question as to whether, for three voters and two issues, their finagle point is in the majority vote yolk; it is.

\section{Selective finesse points and a selective core}

There are times when certain minimal winning coalitions can be ignored; e.g., it may be that certain coalitions are not politically feasible. Whenever this is true, the $d$-finesse point is inappropriate because it unnecessarily guards against changes that an opponent will not, or cannot, take. Obviously, the more we know about an opponent the better we know which winning coalitions are relevant. So, rather than defining the $d$-finesse point over all minimal winning coalitions, the selective $d$-finesse point is defined, in the natural manner, over a specified set of minimal winning coalitions.

Definition 4 A selective core point is one that cannot be beaten with specified winning coalitions. Similarly, with Euclidean preferences, a selective $d$-finesse point is a point in $E_{d}(\mathcal{C})$ for all specified minimal winning coalitions $\mathcal{C}$ where $d$ is the smallest value allowing such a point to exist.

With Euclidean preferences, the selective core is determined by the intersection of the Pareto sets of all specified winning coalitions. While the core is empty for Fig. 2a, suppose it is not possible to form the coalition $\{1,3\}$. In this case, the "selective core point" is determined by the two remaining coalitions of $\{1,2\}$ and $\{2,3\}$. As 
Fig. 5 Polarized setting

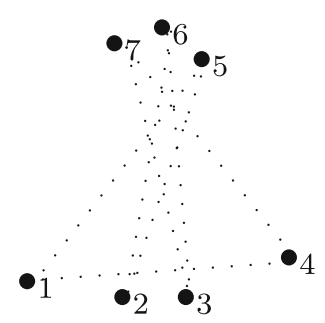

$\mathcal{P}(\{1,2\}) \cap \mathcal{P}(\{2,3\})=\{2\}$, which is voter two's ideal point, this point is the selective core point.

Adding an issue, maybe a divisive one, could separate old coalitions or create new ones; the new issue may even be a strategic move to replace the original core with a selective core or finesse point. This dynamic is depicted in Fig. 5 where, had there been one issue on the $x$-axis, the core would be voter six's ideal point with voters one and four widely separated and never in the same minimal winning coalition. The new issue, depicted by the $y$-axis, is sufficiently divisive to make $\{1,2,3,4\}$ a natural minimal winning coalition. While the core does not exist, if the setting is sufficiently polarized so that $\{1,2,3,4\}$ and $\{5,6,7\}$ are the only realistic coalitions, then $\mathcal{P}(\{1,2,3,4\})$ is the selective core. If the realistic minimal winning coalitions expand to involve any four of voters $\{1,2,3,4,5\}$, the selective core moves to a position near the median of the dominating majority party. But if the winning coalitions consist of $\{1,2,3,4\},\{2,5,6,7\}$ and $\{3,5,6,7\}$, then a selective core vanishes to be replaced by the selective $d$-finesse point; it is close to the median of $\mathcal{P}(\{1,2,3,4\})$.

The following result is immediate.

Theorem 7 For q-rule and a specified selection of minimal winning coalitions, with any number of voters and issues, the following are true.

1. The q-rule selective $d$-finesse point exists. In general, the $d$ value for a selective $d$-finesse point is smaller than that for a $d$-finesse point.

2. A point is a q-rule selective $d$-finesse point for $d=0$ if and only if the point is a selective q-rule core point.

3. A selective q-rule $d$-finesse point is the selective $q$-rule finagle point and the q-rule malicious point. The maximum amount of change in a position needed to respond to the opponent, with either positive or negative campaigning, is $d$.

\section{Conclusion}

It is informative to compare what happens with voting dynamics with results from general dynamics. Voting dynamics linked to a core, or a selective core, force each winning proposal to be closer to the core. Notice how this dynamic resembles "asymptotic stability" from physics where a particle continually moves toward an equilibrium. Physics also uses "orbital stability" where points may not be attracted to a stable point but they remain nearby. Our $d$-finesse point corresponds to a controlled system approach to orbital stability in voting. 
The $d$-finesse point, which extends the core while refining the yolk, is a partial response to the negativity of the chaos theorem. The chaos theorem asserts that anything can happen; the $d$-finesse point identifies a centrally located position that ensures at least a level of stability by exercising appropriate responses. In spatial voting, adding issues tends to generate instabilities (Saari 2004). On the other hand, introducing appropriate issues may create a sense of stability by making certain winning coalitions ineffective. The associated stability is captured with the selective core and $d$-finesse points.

\section{Appendix}

Proof of Theorem 2 With each candidate in the Pareto set, the line connecting a candidate's position with the nearest point on a winning set passes through the swing voter's ideal point. Let $r_{i, j}$ be the radius of the circle centered at voter $j$ 's ideal point and passing through candidate $i$ 's position. The circle geometry of Euclidean preferences requires that, for positive campaigning, $\left|r_{C, j}-r_{O, j}\right|$ is the shortest distance to move Candidate's position to the leaf edge of the circle defined by Opponent's position and voter $j$ 's ideal point. The closest strategy is identified by the smallest value of $\left\{\left|r_{C, 1}-r_{O, 1}\right|,\left|r_{C, 2}-r_{O, 2}\right|,\left|r_{C, 3}-r_{O, 3}\right|\right\}$. A similar argument for negative campaigning shows that $\left|r_{O, j}-r_{C, j}\right|$ is the shortest distance to move Opponent's position to the leaf edge defined by Candidate's position and voter $j$ 's ideal point. As the smallest distance for both possibilities uses the same set of numbers, the conclusion follows. This completes the proof.

Proof of Theorem 3 Part 1. With a finite number of voters, the Pareto set over all voters (defined by their ideal points) is bounded. Thus for a sufficiently large value of $\mathrm{d}$ and any winning coalition $\mathcal{C}$, set $E_{d}(\mathcal{C})$ includes the Pareto set. For this $d$ value, all $E_{d}(C)$ sets intersect. It now follows from standard analysis and the compactness of the $E_{d}(C)$ sets that a minimal $d$ value exists where all $E_{d}(\mathcal{C})$ sets still meet. Such an intersection point is a $d$-finesse point.

Part 2. Point $\mathbf{p}$ is a core point iff $\mathbf{p}$ is in each winning coalition's Pareto set $\mathcal{P}(\mathcal{C})$ iff $\mathbf{p} \in E_{0}(\mathcal{C})$ for each minimal winning coalition $\mathcal{C}$ iff $\mathbf{p}$ is a $d$-finesse point for $d=0$.

Part 3. At a $d$-finesse point, the largest possible distance from Opponent's position to the boundary of the winning set is $d$. That boundary is created by a circle (or sphere) passing through Candidate's current position with center at a voter's ideal point. By moving Candidate's position $d$ units directly toward that voter's ideal point, the new circle, defining the new winning set, passes precisely through Opponent's position. As such a move changes Candidate's position, this is positive campaigning. According to the definition of a $d$-finesse point, if Candidate's position differs from a $d$-finesse point, Opponent could find position requiring Candidate to change by more than $d$ units.

Similarly, with negative campaigning, Candidate must move Opponent's perceived position outside of the winning set. If Candidate is located at the $d$-finesse point, then the largest possible distance is $d$ - the largest possible distance in a winning set. However, if Candidate's position is not at the $d$-finesse point, then, from the definition of 
the $d$-finesse point, Opponent can find positions that would require a larger change in her position to move out of the winning set. Thus a $d$-finesse point is a malicious point.

Proof of Theorem 4 By construction, at the finesse point we have $r_{i}+r_{k}=z_{i, k}+2 d$ and $r_{i}+r_{j}=z_{i, j}+2 d$. Subtracting the first from the second yields Eq. 2 . To derive Eq. 3, adding $r_{1}+r_{2}=z_{1,2}+2 d$ to the Eq. $2 r_{1}-r_{2}=z_{1,2}-z_{1,3}$ leads to $2 r_{1}=z_{1,2}+z_{1,3}-z_{2,3}+2 d$. Solving for $d$ and expressing the equation in a general form yields $d=r_{j}-\frac{1}{2}\left[z_{j, k}+z_{j, i}-z_{k, i}\right]$; this is the first part of Eq. 3. Adding the three equation gives $3 d=\sum r_{j}-\frac{1}{2} \sum_{j<k} z_{j, k}$, or the second part of Eq. 3 .

Finding the finesse point for all $q=2$ three-voter examples. This constructive approach is based on the material introduced just after Def. 1 where the $d$-ellipse for coalition $\mathcal{P}(\{i, j\})$ is defined by points of intersection of the circles of radius $r_{i}=\rho_{i}+d$ and $r_{j}=\rho_{j}+d$ where

$$
\rho_{i}+\rho_{j}=z_{i, j}
$$

Recall, each pair of circles defines two points on the $d$-ellipse; the relevant part of the $d$-ellipse is found by varying the $\rho_{i}$ value from 0 to $z_{i, j}$ so that all points on the line interval are covered.

A finesse point fixes the $r_{j}$ and $d$ values. An important observation is that, according to $r_{j}=\rho_{j}+d$, the finesse point also fixes the $\rho_{j}$ values that are associated with this finesse point, and the values are the same for both coalitions to which $j$ belongs. The importance of having fixed $\rho_{j}$ values for both coalitions is that the values can be determined with elementary algebra. Namely, it follows that

$$
\rho_{1}+\rho_{2}=z_{1,2}, \quad \rho_{1}+\rho_{3}=z_{1,3}, \quad \rho_{2}+\rho_{3}=z_{2,3}
$$

the solution for this system of equations is

$$
\rho_{1}=\frac{z_{1,2}+z_{1,3}-z_{2,3}}{2}, \quad \rho_{2}=\frac{z_{1,2}+z_{2,3}-z_{1,3}}{2}, \quad \rho_{3}=\frac{z_{1,3}+z_{2,3}-z_{1,2}}{2} .
$$

Thus, $\rho_{j}$ is half the sum of the lengths of the two adjoining legs minus the length of the third leg (See Eq. 3).

To find the $d$ value and the finesse point for a given configuration of three ideal points, just solve the three equations coming from these $\rho_{j}$ values and the $r_{k}=\rho_{k}+d$ expressions. Namely, if the $k$ th agent's ideal point is located at $\left(p_{1}, p_{2}\right)$, then the associated equation is $\left(x-p_{1}\right)^{2}+\left(y-p_{2}\right)^{2}=\left(\rho_{k}+d\right)^{2}$.

To illustrate, suppose the first, second, and third voters' ideal points are located, respectively, at $(0,0),(5,0)$, and $(0,12)$, so $z_{1,2}=5, z_{1,3}=12, z_{2,3}=\sqrt{5^{2}+12^{2}}=13$. From these $z_{i, j}$ values and Eq. 8, it follows that $\rho_{1}=2, \rho_{2}=3, \rho_{3}=10$.

If the finesse point is located at $(x, y)$, then the three equations in the three unknowns are obtained by expressing the $r_{k}^{2}=\left(\rho_{k}+d\right)^{2}$ values in the above algebraic form: 


$$
x^{2}+y^{2}=(2+d)^{2}, \quad(x-5)^{2}+y^{2}=(3+d)^{2}, \quad x^{2}+(y-12)^{2}=(10+d)^{2} .
$$

Solving these equations leads to the values

$$
x=2-\frac{d}{5}, \quad y=2-\frac{2 d}{3}, \quad d=0.13276 \quad \text { or } \quad x=1.97345, \quad y=1.9115 .
$$

Consistent with the Sect. 4 description about the finesse point, notice how the $d$ value is quite small, about 0.026 the shortest $z_{j, k}$ length, and how the finesse point is located near the ideal point with the shortest legs (i.e., near the juncture of the two minimal winning coalitions with the stronger ties). While this algebraic approach holds for all three-voter, two issue, majority vote configurations, the above example was selected to minimize the occurrence of square roots.

A similar construction holds for any $q$-rule setting with any number of issues. However, the number of equations that must be used increases the complexity of the analysis. With $q=4$ from Fig. $4 \mathrm{a}$, for instance, the one $\left(x-p_{1}\right)^{2}+\left(y-p_{2}\right)^{2}=\left(\rho_{k}+d\right)^{2}$ for a pair of points is replaced by six equations involving all pairs of points. Other geometric analysis, however, can reduce the number of necessary equations.

Proof of Theorem 5 The leg length inequalities of Eq. 4 follow from Eq. 2. From the law of cosines, $\cos \left(\phi_{j, k}\right)=\frac{r_{j}^{2}+z_{j, k}^{2}-r_{k}^{2}}{2 r_{j} z_{j, k}}$. As larger cosine values correspond to smaller angles, the Eq. 4 angle inequalities follow from the inequalities on leg lengths and the $r_{j}$ values.

Proof of Eq. 5 An ellipse with both foci (the nails in the description) on the $x$-axis, $z$ distance apart, and $x=0$ in the center, has the equation $\frac{x^{2}}{a^{2}}+\frac{y^{2}}{b^{2}}=1$ for some $a>b>0$ values. When $y=0$, the $d$-ellipse is at point $x=\frac{z}{2}+d$; this is the $a$ value. When $x=0$, the string defining a $d$-ellipse forms an isosceles triangle, where the height above the $x=0$ point is

$$
\sqrt{\left(\frac{z}{2}+d\right)^{2}-\left(\frac{z}{2}\right)^{2}}=\sqrt{z d+d^{2}}=d \sqrt{1+\frac{z}{d}}
$$

this is the $b$ value. Notice that this $2 b$ width yields Eq. 5. Other properties follow from the $d$-ellipse's equation, $\frac{x^{2}}{((z / 2)+d)^{2}}+\frac{y^{2}}{z d+d^{2}}=1$. For instance, a circle of diameter $2 d$ with center anywhere between the two ideal points is in the $d$-ellipse; e.g., the ellipse width at an ideal point is $2 d\left[2-\frac{1}{1+\frac{z}{2 d}}\right]>2 d$.

Proof of Theorem 6 (Parts 1, 5) To show for $n=3$ that the finagle and finesse points agree, we outline the construction of the finagle point (see Wuffle et al. (1989) for more details.) Find three circles (Fig. 6a) where each is centered at a voter's ideal point and tangent to the other two. Such circles exist and are uniquely determined. (In general, such tangencies do not exist, so this is what limits the finagle point approach.) 
Fig. 6 The yolk versus the finesse point. a Finagle point, b $E_{d}^{2}(C)$ suffices for two issues

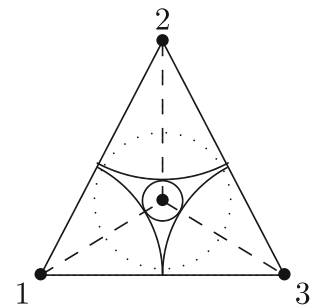

(a)

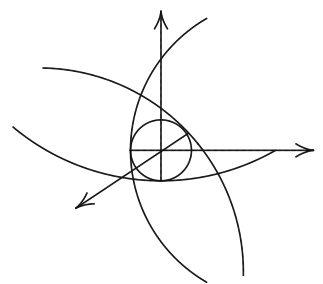

(b)

In the curvilinear triangle defined by the three circles, find the largest inscribed circle: the finagle point is the circle's center.

To prove that this point is our majority vote $d$-finesse point, let $r$ be the radius of the circle centered at the finagle point. The dashed lines connecting any two ideal points is the distance between the two ideal points plus $2 r$, so it is on a $r$-ellipse. As this is true for all pairs of ideal points, the finagle point is a $d$-finesse point where $r=d$. That this is the minimum $d$ value follows from the fact that in the Pareto set, the three $d$-ellipses have a unique point of intersection. While the finagle point is our $d=r$ finesse point for $n=3$, our construction does not rely on this special Fig. 6a behavior, which holds primarily for triangles and special symmetric figures: this is what permits our $d$-finesse point to hold for any number of voters, issues, and $q$-rules.

To show for $n=3$ that $\mathbb{S}_{d}(2)$ is inside $\mathbb{Y}(2)$, notice that $\mathbb{Y}(2)$ touches all three edges of the triangular Pareto Set (in Fig. 6a, $\mathbb{Y}(2)$ is given by the dotted circle). Each ideal point is on two edges and $\mathbb{Y}(2)$ is tangent to each triangle edge, so the two distances from an ideal point to the two tangent points are equal. If $\rho_{j}$ is the common distance from ideal point $i$, then $\rho_{1}+\rho_{2}=z_{1,2}, \rho_{1}+\rho_{3}=z_{1,3}, \rho_{2}+\rho_{3}=z_{2,3}$. The locations of the tangent points follow from these three equations with three unknowns. These tangent points also define the three Fig. 6a circles, so $\mathbb{S}_{d}(2)$ is in the $\mathbb{Y}(2)$ interior. Moreover, the lines from the $\mathbb{Y}(2)$ center to these tangent points are perpendicular to the edges and tangent to the two $\rho_{j}$ circles. As these perpendicular lines cross at the center of the yolk and also intersect (by construction) $\mathbb{S}_{d}(2)$, the yolk's center is in $\mathbb{S}_{d}(2)$.

To show that $\mathbb{S}_{d}(n-1) \subset \mathbb{Y}(n-1)$ with $(n-1)$ issues where the $n$-ideal points define a nondegenerate $n$-simplex, note that $\mathbb{Y}(n-1)$ is tangent to the $n$ planes defined by $(n-1)$ ideal points; each ideal point is in $n-1$ hyperplanes. As $\mathbb{Y}(n-1)$ is a sphere, the line in each hyperplane from the $j$ th ideal point to the $\mathbb{Y}(n-1)$ tangent point has the same length $\rho_{j}$. Thus, in any hyperplane, the $\rho_{j}$ values define a unique point-the $\mathbb{Y}(n-1)$ tangent point. About the $j$ th ideal point, construct a sphere of radius $\rho_{j}$. As there are precisely $n$ of these spheres, a unique sphere can be constructed in Pareto set that is tangent to each of $n$ constructed spheres. This is $\mathbb{S}_{d}(n-1)$, by construction it is in $\mathbb{Y}(n-1)$. Also by construction, the line perpendicular to each hyperplane meets the center of $\mathbb{Y}(n-1)$ and is tangent to each of the $(n-1)$ constructed spheres, so it is in $\mathbb{S}_{d}(n-1)$. As this is true for all such lines, the yolk center is in the adjustment set.

The proof for $\mathbb{S}_{d}(q)$ and $\mathbb{Y}(q), \frac{n}{2}<q<n$, involves only minor changes; e.g., each ideal point is in $\left(\begin{array}{l}n-1 \\ q-1\end{array}\right)$ minimal coalitions and each coalition's Pareto set has dimension 
$q-1$. At least $n-1$ of them are tangent to $\mathbb{Y}(q)$, while others may intersect this yolk. The construction using these tangent points and spaces is the same as for $q=(n-1)$ except instead of $(n-1)$ choices of $\rho_{j}$ in each $(q-1)$-dimensional hyperplane, there are $q$ of them, and the orthogonal line from each of these hyperplanes is replaced with a $(n-1)-(q-1)$ dimensional orthogonal hyperplane.

(Part 2.) To prove for $n=3$ that the $d$-finesse point is at the $\mathbb{Y}(2)$ center iff the Pareto set is an equilateral triangle, notice that the $\mathbb{Y}(2)$ center is at the intersection of the angle bisectors of the Pareto set's angles. Thus, according to Eq. 4, the $d$-finesse point is at the $\mathbb{Y}(2)$ center iff $\phi_{j, k}=\phi_{j, i}$ for all $j$, which (Theorem 5) requires all leg lengths of the Pareto set to agree-it is an equilateral triangle. To show that the $q$-rule finesse point generally is not at the $\mathbf{Y}(q)$ center, consider a Pareto set that is a $k$-dimensional simplex with $q$-hyperplanes as the faces of the simplex. With the above construction, construct the finesse point in terms of the tangent points of the yolk on the hyperplanes. If the simplex is not equilateral, some radii are longer than others, so the inscribed sphere cannot have its center at the $\mathbb{Y}(q)$ center.

(Part 3.) For $\frac{n}{2}<q_{1}<q_{2}$, any minimal winning coalition for a $q_{1}$ rule, $\mathcal{C}_{1}$, is contained in some minimal winning coalition for a $q_{2}$ rule, say $\mathcal{C}_{2}$. Thus $E_{d}\left(\mathcal{C}_{1}\right) \subset E_{d}\left(\mathcal{C}_{2}\right)$, so the $E_{d}(\mathcal{C})$ sets for the $q_{2}$ rule cannot meet at a larger $d$ value than those for the $q_{1}$ rule.

(Part 4.) To show that a $q$-rule finesse point $\mathbf{p}$ is a $q$-rule uncovered point, suppose $\mathbf{p}_{1}$ beats $\mathbf{p}$; i.e., $\mathbf{p}_{1}$ is in the winning set defined by $\mathbf{p}$. As $\mathbf{p}_{1}$ is not a finesse point, the winning set it defines for some coalition is larger than that defined by $\mathbf{p}$. Consequently, there are points that beat $\mathbf{p}_{1}$ that do not beat $\mathbf{p}$; thus $\mathbf{p}$ is an uncovered point.

Width of winning set. In defining the finesse point, we claimed that $E_{d}^{2}(\mathcal{C})$ suffices for two issues. First, place the largest possible ball in the winning set (Fig. 6b). The ball is tangent to circles about ideal points, so a vector orthogonal to the ball is orthogonal to the circle: it points toward the center of the ball and the particular ideal point. If the ball is not at the center of a lens defined by two circles, then some circle on one side holds it above the lens' center. The two orthogonal vectors for the lens have components toward the lens' center, while the third one points away. Thus, with two dimensions, the convex hull of the three ideal points must include the ball. As the ideal points are in coalition $\mathcal{C}$, for the winning set to have a ball, the point $\mathbf{p}$ must be in a third dimension; i.e., there are more than two issues. Thus the winning set's width for two issues is given by the lens width of two circles. The first ellipse from $E_{d}^{2}(\mathcal{C})$ that meets $\mathbf{p}$ captures the smallest width of lens defined by $\mathbf{p}$, so the $d$ value captures the winning set's width.

A similar argument shows that with $k$ issues, the largest ball in a winning set is bounded by at most $k$ circles; the width of this structure is captured by the $k$-fold $d$-ellipsoid. Some $\mathbf{p}$ choices may allow the lens of $j$ spheres where $j<k$, to define the winning set's width. To handle such situations, $E_{d}(\mathcal{C})$ is defined as the union of the $E_{d}^{j}(\mathcal{C})$ sets for $j=2, \ldots, k$.

Proof of Theorem 7 The proofs follow that of the earlier theorems but over a subset of the winning coalitions. Thus, for instance, the minimum values for $d$ are equal or smaller. 
Open Access This article is distributed under the terms of the Creative Commons Attribution Noncommercial License which permits any noncommercial use, distribution, and reproduction in any medium, provided the original author(s) and source are credited.

\section{References}

Downs A (1957) An economic theory of democracy. Harper and Row, New York

Ferejohn JA, McKelvey RD, Packel EW (1984) Limiting distributions for a continuous state Markov models. Soc Choice Welf 1(1):45-67

Hotelling H (1929) Stability and competition. Econ J 39(March):41-57

McKelvey R (1979) General conditions for global intransitivities in formal voting models. Econometrica 47(September):1085-1112

McKelvey R (1986) Structural instability of the core. J Math Econ 15(3):179-198

Miller NR (1980) A new solution set for tournament and majority voting. Am J Polit Sci 24:68-96

Miller NR (2005) The effects of clustered ideal point configurations on the location and size of the yolk. http://research.umbc.edu/ nmiller/RESEARCH/CLUSTERS.FIN.htm

Nurmi H (2002) Voting procedures under uncertainty. Springer, New York

Richards D (1994) Intransitivities in multidimensional spatial voting. Soc Choice Welf 11(2):109-119

Saari DG (1995) Basic geometry of voting. Springer, New York

Saari DD (2004) Geometry of stable and chaotic discussion. Am Math Monthly 111:377-393

Saari DG (2007) Hidden mathematical structures of voting. In: Simeone B, Pukelsheim F (eds) Mathematics and democracy: recent advances in voting systems and collective choice. Springer, New York, pp 221-234

Shepsle K, Weingast B (1984) Uncovered sets and sophisticated voter outcomes with implications for agenda institutions. Am J Polit Sci 28:49-74

Stone R, Tovey C (1992) Limiting median lines do not suffice to determine the yolk. Soc Choice Welf 9:33-35

Tataru M (1996) Growth rates in multidimensional spatial voting. Ph.D. Dissertation, Northwestern University

Tataru M (1999) Growth rates in multidimensional spatial voting. Math Soc Sci 37:253-263

Wuffle A, Feld S, Owen G, Grofman B (1989) Finagle's Law and the Finagle Point, a new solution concept for two-candidate competition in spatial voting games without a core. Am J Polit Sci 33:348-375 